\title{
Characterization of an Air Jet Haptic Lump Display
}

\author{
Matteo Bianchi, James C. Gwilliam, Alperen Degirmenci, and Allison M. Okamura
}

\begin{abstract}
During manual palpation, clinicians rely on distributed tactile information to identify and localize hard lumps embedded in soft tissue. The development of tactile feedback systems to enhance palpation using robot-assisted minimally invasive surgery (RMIS) systems is challenging due to size and weight constraints, motivating a pneumatic actuation strategy. Recently, an air jet approach has been proposed for generating a lump percept. We use this technique to direct a thin stream of air through an aperture directly on the finger pad, which indents the skin in a hemispherical manner, producing a compelling lump percept. We hypothesize that the perceived parameters of the lump (e.g. size and stiffness) can be controlled by jointly adjusting air pressure and the aperture size through which air escapes. In this work, we investigate how these control variables interact to affect perceived pressure on the finger pad. First, we used a capacitive tactile sensor array to measure the effect of aperture size on output pressure, and found that peak output pressure increases with aperture size. Second, we performed a psychophysical experiment for each aperture size to determine the just noticeable difference (JND) of air pressure on the finger pad. Subject-averaged pressure JND values ranged from 19.4-24.7 kPa, with no statistical differences observed between aperture sizes. The aperture-pressure relationship and the pressure JND values will be fundamental for future display control.
\end{abstract}

\section{INTRODUCTION}

Distributed tactile information is important in haptic exploration tasks, such as detecting hard lumps in soft tissue. However, tactile feedback is not provided by existing clinical robot-assisted minimally invasive surgical (RMIS) systems. Few studies have demonstrated tactile displays capable of integration within an RMIS system, largely because of the size and weight constraints required to fit the display within the small space of the master manipulators [1]. Our longterm goal is to create a tactile lump display for use in RMIS, capable of rendering the perception of a small lump to the fingertip. While many tactile displays described in the literature are designed to recreate a local surface profile through an array of mechanically actuated pins (e.g., [2],

This work was supported in part by the US Army Medical Research and Material Command under Contract No. W81XWH-11-C-0050, a National Science Foundation Graduate Research Fellowship, and the European Commission Collaborative Project no. 248587, "THE Hand Embodied", within the FP7-ICT-2009-4-2-1 program "Cognitive Systems and Robotics".

The views, opinions and/or findings contained in this report are those of the author(s) and should not be construed as an official Department of the Army position, policy or decision unless so designated by other documentation.

M. Bianchi is with Interdepartmental Research Center "E. Piaggio", University of Pisa, via Diotisalvi 2, 56126 Pisa, Italy matteo.bianchi@centropiaggio.unipi.it

J.C. Gwilliam, A. Degirmenci, and A.M. Okamura are with the Laboratory for Computational Sensing and Robotics, Johns Hopkins University, Baltimore, MD 21218, USA \{jim.gwilliam, alperen, aokamura\} ejhu.edu

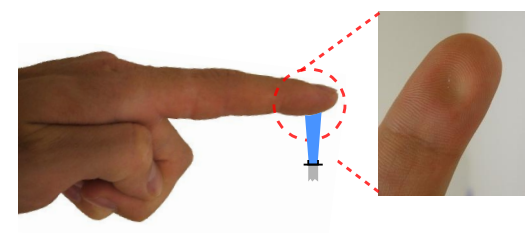

(a) Lump display concept

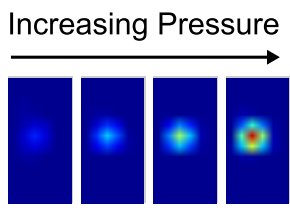

(b) Tactile sensor output

Fig. 1. Proposed lump display method and output measurement. (a) A thin jet-stream of air is directed to the finger pad, causing the skin to indent in a hemispherical shape and eliciting the percept of a lump. (b) Capacitive tactile sensor array is used to measure the output pressure of the display as aperture size and supply pressure are varied.

[3], [4]), the size and weight constraints of RMIS motivate a more targeted approach. We use a pneumatic technique that has been demonstrated but not rigorously tested [5]. We generate the percept of feeling a lump by directing a narrow jet-stream of air to the finger, which deforms the skin of the finger pad in a hemispherical pattern (Fig. 1a).

We expect that we can control the perceived size and stiffness of a lump felt at the finger pad by regulating (1) airjet aperture size and (2) supply pressure. In this work, supply pressure refers to the pressure "supplied" to the display, and output pressure refers to the pressure delivered to the finger pad. However, there is a complex relationship between aperture size, supply pressure, and the resulting perceived output pressure on the finger pad, which we address in this work through measurements and psychophysical experiments.

Ideally, the display output pressure would be determined using models of fluid dynamics. However, an accurate model is difficult to obtain since the system is affected by many factors, including air temperature, the type of air flow (e.g., laminar, turbulent), and resistive pressure losses due to friction along the length of the tubing [6]. In this study we adopt an empirical strategy to quantify the output pressure using tactile sensors. We measure peak tactile sensor pressure values across a range of supply pressures and aperture sizes (Fig. 1b). This empirical data will be useful to characterize the output pressure of the display as the aperture size varies, and can be used as a feed-forward control in future versions of the display.

We also use a classic psychophysical method (constant stimuli) to find the just noticeable differences (JND) of supply pressure for each aperture size. This study will establish the necessary changes in supply pressure for each aperture size to elicit a perceived change in the output pressure. This paper evaluates the proposed display method and provides proof-of-concept experiments, laying a foundation for the development of new tactile displays. 


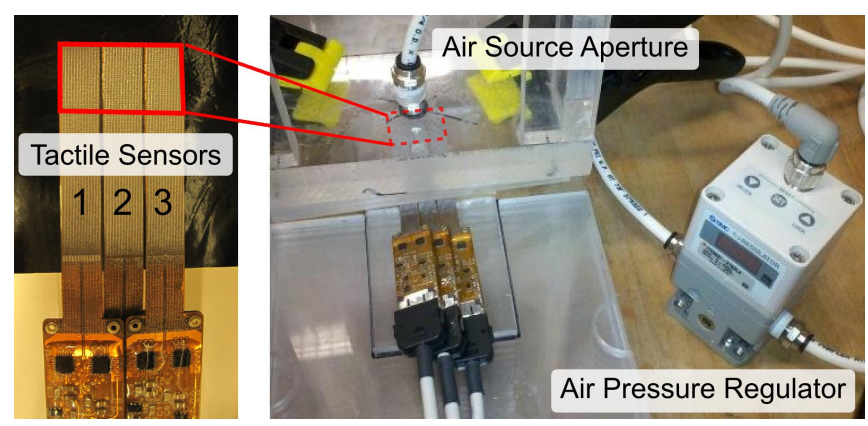

Fig. 2. Setup for tactile sensor measurement. The air pressure regulator connects directly to the air source aperture and controls supply pressures. Tactile sensors placed under the aperture record distributed pressure as supply pressure and aperture size are varied.

\section{Materials AND Methods}

\section{A. Tactile Sensor Experiment}

The experimental apparatus measures distributed pressure patterns on a tactile sensor array while air supply pressure and outlet aperture size are controlled. The apparatus (Fig. 2) consists of three main components: (1) An electronically controlled air pressure regulator, (2) a set of interchangeable aperture diameters through which the air escapes, and (3) a tactile sensor array to measure the output.

The electronically controlled pneumatic regulator (SMCITV2031-21N2L4, SMC Corporation, Noblesville, IN, USA) provides step-less control of air pressure (0.05-0.5 MPa) proportional to an electrical signal $(0-5 \mathrm{~V})$, with a maximum flow rate of $1500 \mathrm{~L} / \mathrm{min}$. Linearity between the input signal and set pressure is within $\pm 1 \%$ full scale (F.S.), hysteresis within $\pm 0.5 \%$ F.S. and sensitivity within $0.2 \%$ F.S.

The aperture size was set with six acrylic plates $(5.4 \mathrm{~mm}$ thick), each with a single drilled hole diameter ranging from $1.0-2.5 \mathrm{~mm}$ in $0.3 \mathrm{~mm}$ increments. A plate was clamped to the underside of the apparatus with the aperture centered over the air source aperture, separated by a rounded rubber washer to prevent air leaks. The regulator-supplied air was then forced through the fixed aperture onto the tactile sensors.

The tactile sensor array is composed of three smaller sensors (DigiTacts, Pressure Profile Systems, Los Angeles, CA, USA) arranged contiguously to form a $6 \times 12$ array of tactile sensing elements, occupying a total footprint of $12 \times 25 \mathrm{~mm}$ (Fig. 2, left). Each individual sensor contains 24 sensing elements (each element measuring $1.8 \times 1.8 \mathrm{~mm}$ ). The sensors are capacitive and provide a linear response with a sensing range of $0-0.14 \mathrm{~N} / \mathrm{mm}^{2}$ and a sensitivity of $6.9 \times 10^{-4} \mathrm{~N} / \mathrm{mm}^{2}$.

A graphical user interface was developed in the QT environment (QT Creator, Nokia, Helsinki, Finland) to send pre-calibrated voltage levels from the computer via a NIDAQ card (DAQCard-6024E, National Instruments, Austin, TX, USA) to the electronic pressure regulator. The tactile sensor array was centered $14 \mathrm{~mm}$ below the output aperture on a flat rigid surface. Prior to each measurement, the tactile sensor array was set to a zero baseline to remove any residual pressures and increase the signal-to-noise ratio (SNR) of

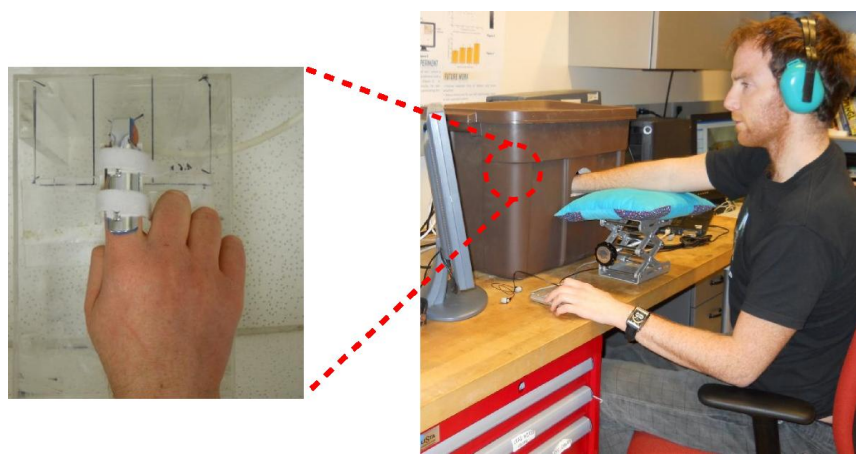

Fig. 3. Setup for psychophysical experiment. A subject's right index finger is fixed in place $14 \mathrm{~mm}$ above the aperture. Subjects enter responses about paired stimuli using a numerical keypad with the left hand. The display apparatus is placed within a sound-reducing box and subjects wear in-ear headphones playing white noise and over-ear protection to prevent auditory cues.

the measurement. Seven discrete and equally spaced supply pressure levels between 10 and $70 \mathrm{psi}(68.9-482.6 \mathrm{kPa})$ were used to output air from the display onto the sensor array and each was recorded for 30 seconds. Each set pressure level was evaluated two times, first in ascending, then descending order, to allow for hysteresis analysis. Data for each 30second measurement were averaged for each sensing element to create a single array of pressure values, hereafter referred to as a "tactile image" (e.g. Fig. 1b). This procedure was repeated for each aperture size.

\section{B. Human Psychophysics Experiment}

1) Subjects: Ten healthy right-handed volunteers (5 male, 5 female, ages 20-28) gave informed consent to perform the psychophysical experiment. No subjects had physical limitations that would affect the experimental outcomes. Data collection from subjects in this study was approved by the Johns Hopkins University Homewood Institutional Review Board.

2) Experimental Task: The aim of this experiment was to determine the air supply pressure JND for each aperture size. Subjects were seated comfortably with the right index finger fixed in a finger holder, palm down, with the center of the distal finger pad centered over the aperture (Fig. 3, left). A skin-safe adhesive tape (Trutape LLC, USA), was applied to the back of the index finger distal and middle phalanges to restrict any movement of the distal interphalangeal joint. An additional finger brace was fastened around the back side of the finger to prevent motion of the proximal interphalangeal joint. Securing the finger in this manner prevents the introduction of unwanted experimental biases. The distance from the exposed finger pad to the aperture was fixed at 14 $\mathrm{mm}$, consistent with the distance used in the tactile sensor experiment. The resulting JND is likely dependent on this distance.

Subjects received air-jet pressure to the finger pad in the form of separate but paired supply pressure stimuli, and they were asked to indicate which stimulus in the pair produced the larger output pressure percept. Each pair consisted of 
a reference stimulus (RS) of $45 \mathrm{psi}(310.3 \mathrm{kPa})$ and a comparison stimulus (CS), presented in random order. We used seven equally spaced comparison stimuli ranging from 30 to $60 \mathrm{psi}(206.8-413.7 \mathrm{kPa})$. The minimum and maximum supply pressure levels used were chosen in a preliminary study such that they were almost always judged as less than or greater than the RS, respectively [7].

The experiment consisted of five sub-parts, one for each aperture size $(1.3,1.6,1.9,2.2$, and $2.5 \mathrm{~mm})$. The order of aperture sizes tested was randomized for each subject. An aperture plate was fixed to the apparatus in the same manner as described in the tactile experiment (Section IIA). A single trial consisted of: the first stimulus $(150 \mathrm{~ms})$, an inter-stimuli interval $(50 \mathrm{~ms})$, and the second stimulus $(150 \mathrm{~ms})$, followed by the subject's response. An external monitor displayed "1" or "2" during the presentation of the first or second stimulus, respectively, followed by "Respond Now" at the conclusion of paired stimuli. Subjects then indicated the greater pressure stimulus ("1" or "2") using an external numerical keypad, which was stored and written to a file. Subjects were permitted to experience a particular paired stimuli as many times as necessary by inputting an invalid response, which would repeat the previous paired stimuli. The procedure was automated, such that a subject's response automatically initiated the next trial using the randomly pregenerated stimulus set. Each sub-part consisted of 168 paired stimuli presented randomly ( 7 stimulus levels $\times 24$ pairwise discriminations), and lasted approximately 15 minutes, with at least a two-minute break afterward. For all trials, subjects wore in-ear headphones playing white noise and over-ear hearing protection to prevent auditory cues (Fig. 3, right). Prior to the experiment, subjects were trained briefly using manually selected pressure pairs. These responses were not recorded.

3) Analysis: A subject's response proportion $(P)$ was computed for each stimulus level and expressed as $P=$ $\sum y_{i} / n$, where $y_{i}=1$ if the comparison stimulus (CS) was perceived as greater than the reference stimulus (RS), and $y_{i}=0$ otherwise, and $n$ is the number of pairwise discriminations performed for each CS. In trials where the RS was compared against itself, we randomly predefined the first stimulus as the RS in half of the trials, and the second stimulus in the other half of trials.

To determine JND values, a psychometric function was constructed for each set of data points. Gesheider [7] suggests that if a sufficient number of measurements are obtained in a psychophysical experiment, the resulting psychometric function will often resemble an ogive curve. We tested the ogive assumption (and in the process inferred an initial qualitative assessment of our data) by determining whether a linear relationship existed between the z-scores of the response proportion values $(P)$ and the corresponding stimulus intensities. Z-scores were obtained by computing the inverse of the cumulative distribution function for the normal distribution, evaluated at the levels of $P$. Linearity was assessed using the method of least squares, and is described by the resulting $R^{2}$ value (coefficient of determination).

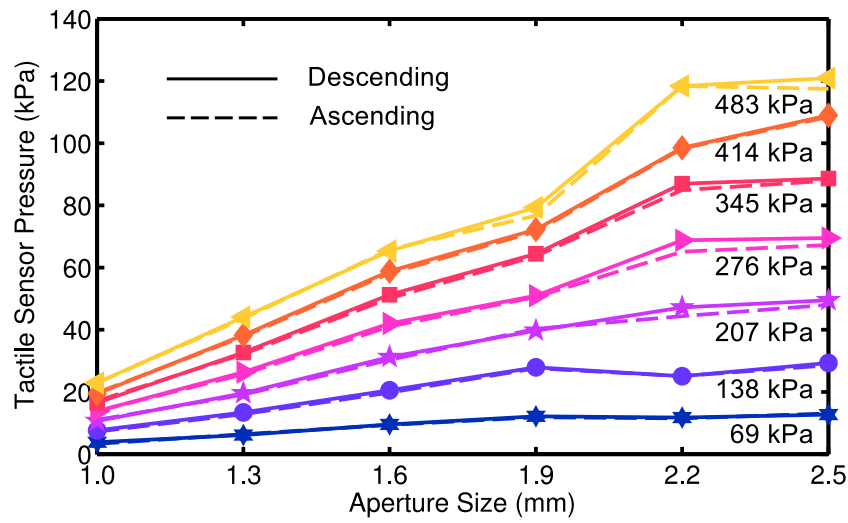

Fig. 4. Tactile sensor measurement results. Each data point represents the peak output pressure value measured on the tactile sensor array for a particular air supply pressure and aperture size. Numbers at right represent supply pressures (rounded). Line styles indicate order of supply pressures used in measurement.

A psychometric function was fit to each data set using the psignifit toolbox version 2.5.6 for Matlab (http://bootstrapsoftware.org/psignifit/), with the default logistic function, which implements the maximum-likelihood method described by Wichmann and Hill [8]. The psignifit toolbox was also used to evaluate the goodness of fit for each curve in terms of deviance and deviance residuals.

\section{RESUltS AND DISCUSSION}

\section{A. Tactile Sensor Measurements}

Peak pressure values represent the maximum pressure observed on the tactile image and are shown for all aperture sizes and supply pressures in Fig. 4. Peak pressures from the tactile images increase with aperture size. This occurs in part because larger apertures exhibit a decreased resistance to air flow. Additionally, the Venturi effect suggests that a reduction in fluid pressure occurs when a fluid flows through a constricted section (aperture) [6].

Moreover, peak output pressure values for a given aperture size appear to be almost equally spaced, although this spacing drastically increases for larger apertures sizes. The similarity of the results for ascending and descending supply pressure sequences indicates negligible hysteresis (Fig. 4).

The tactile sensor data gives distributed pressure profiles of the display output. However, this analysis has only considered the peak pressure values of each measurement, which we expect are related to the overall pressure profile. Other metrics, such as profile size and gradient-based analysis may be considered in future analysis.

Fig. 4 also provides a general characterization of the airjet display method. The obtained tactile data prescribe the necessary changes in supply pressure for a given aperture required to achieve a desired outcome, in this case a specific peak output pressure.

\section{B. Psychophysics}

From the method of least squares, the $R^{2}$ values for the psychometric functions were all greater than 0.86 and $86 \%$ 


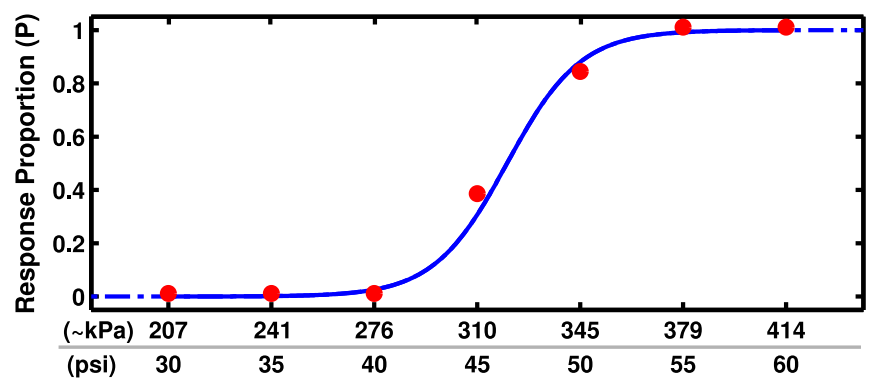

Fig. 5. Psychophysical results for subject 2 using the $2.5 \mathrm{~mm}$ aperture. The points represent the subject's response proportion $(P)$ at each stimulus level. The solid line is the psychometric curve fit to the data using psignifit software.

of $R^{2}$ values were greater than 0.9 , indicating that the ogive assumption is reasonably satisfied [7].

An example subject's response proportion $(P)$ for a given aperture is shown in Fig. 5. The line represents the psychometric function fit to the data. The point of subjective equality (PSE) represents the pressure value on a psychometric curve where $P=0.5$. The PSE should correspond to the value of the RS ( $310.3 \mathrm{kPa})$, which represents the point of objective equality (POE). For each aperture, we averaged PSE values across subjects for whom goodness of fit was obtained for all apertures. PSE values (listed by ascending aperture size) are $308.3 \pm 7.6,310.4 \pm 4.2,307.3 \pm 4.0,313.3 \pm 7.0$, and $310.1 \pm 6.2 \mathrm{kPa}$. All values are close to the POE, indicating a low level of constant error (i.e., difference between PSE and POE). Specifically, the maximum constant error is less than $6 \%$ of the range of supply pressure levels.

To determine the JND for all subjects and aperture sizes, we calculated the lower $\left(D L_{l}\right)$ and upper $\left(D L_{u}\right)$ difference thresholds, which correspond to the pressure ranges between the PSE and the stimulus level where response proportion $(P)$ on the psychometric curve is 0.25 and 0.75 , respectively. JND values were obtained by taking the arithmetic mean of $D L_{l}$ and $D L_{u}$, and are reported in Table I. A mean JND value was computed for each aperture size by averaging JND values across subjects (excluding subjects who lacked goodness of fit on one or more psychometric curves).

We performed a one-factor, within-subjects analysis of variance (ANOVA), which revealed no significant differences in the PSE or JND of air pressure between aperture sizes ( $p=0.3033$ and $p=0.1565$, respectively). This result suggests that the change in supply pressure required to elicit a perceptible change in perceived output pressure is constant, regardless of the aperture size of the display.

\section{CONCLUSIONS AND FUTURE WORKS}

In this work the relationship between aperture size and air supply pressure was investigated by means of tactile sensor measurements and psychophysical experiments, to understand how they affect actual and perceived pressure on the finger pad. Our long term goal is to build an air-jet haptic lump display for use in RMIS, using these two variables to control perceived size and stiffness of the displayed lump.
TABLE I

JND PRESSURE (kPa) FOR EACH SUBJECT AND ALL APERTURES

\begin{tabular}{|c||c|c|c|c|c|}
\hline \multirow{2}{*}{ Subject } & \multicolumn{5}{c|}{ Aperture Diameter } \\
\cline { 2 - 6 } & $\mathbf{1 . 3} \mathbf{~ m m}$ & $\mathbf{1 . 6} \mathbf{~ m m}$ & $\mathbf{1 . 9} \mathbf{~ m m}$ & $\mathbf{2 . 2} \mathbf{~ m m}$ & $\mathbf{2 . 5} \mathbf{~ m m}$ \\
\hline \hline $\mathbf{1}$ & 25.9 & $* 23.0$ & 20.7 & 24.6 & 18.1 \\
\hline $\mathbf{2}$ & 17.3 & 21.2 & 14.6 & 17.0 & 13.5 \\
\hline $\mathbf{3}$ & $* 23.0$ & 20.0 & $* 15.5$ & 19.1 & $* 21.5$ \\
\hline $\mathbf{4}$ & 27.5 & 26.7 & 23.7 & 22.3 & 21.2 \\
\hline $\mathbf{5}$ & 31.7 & 39.2 & 30.9 & 27.0 & 34.5 \\
\hline $\mathbf{6}$ & 27.3 & 17.0 & 14.7 & 14.6 & 18.8 \\
\hline $\mathbf{7}$ & 32.4 & 27.6 & 35.4 & 33.5 & 18.6 \\
\hline $\mathbf{8}$ & 22.5 & 32.3 & 24.2 & 23.9 & 20.9 \\
\hline $\mathbf{9}$ & 16.9 & 17.3 & 11.5 & 6.2 & 18.6 \\
\hline $\mathbf{1 0}$ & 21.9 & 11.5 & 19.8 & 17.3 & 9.4 \\
\hline \hline Mean & $\mathbf{2 4 . 7}$ & $\mathbf{2 4 . 1}$ & $\mathbf{2 1 . 8}$ & $\mathbf{2 0 . 2}$ & $\mathbf{1 9 . 4}$ \\
\hline Std. Dev. & $\mathbf{6 . 0}$ & $\mathbf{9 . 1}$ & $\mathbf{8 . 3}$ & $\mathbf{8 . 3}$ & $\mathbf{7 . 3}$ \\
\hline
\end{tabular}

* Indicates JND obtained from a psychometric curve which did not demonstrate goodness of fit to the response proportion $(P)$ data. Subjects with $*$ are excluded from mean values.

Despite the observed increase in peak output pressure measured by the tactile sensors with increasing aperture size, the psychophysical results suggest that the JND of air pressure on the finger pad is constant, regardless of aperture size. We expect that the JND values found in this study (Table I) will be transferable to a future RMIS-capable display.

Future work includes additional psychophysical experiments to clarify how aperture size and air supply pressure directly affect perceived stiffness and lump size. We will use the tactile data collected in this study to evaluate the size of the air pressure "contact area" on the tactile sensors. This work and future studies will inform the design of, and provide performance specifications for, an RMIS haptic display.

\section{REFERENCES}

[1] A. M. Okamura, "Haptic feedback in robot-assisted minimally invasive surgery," Current Opinion in Urology, vol. 19, pp. 102-107, 2009.

[2] M. V. Ottermo, O. Stavdahl, and T. A. Johansen, "A remote palpation instrument for laparoscopic surgery: design and performance." Minimally Invasive Therapy and Allied Technologies, vol. 18, no. 5, pp. 259-272, 2009.

[3] R. D. Howe, W. J. Peine, D. A. Kantarinis, and J. S. Son, "Remote palpation technology," IEEE Engineering in Medicine and Biology Magazine, vol. 14, no. 3, pp. 318-323, 1995.

[4] J. Killebrew, S. Bensmaia, J. Dammann, P. Denchev, S. Hsiao, J. Craig, and K. Johnson, "A dense array stimulator to generate arbitrary spatiotemporal tactile stimuli," Journal of Neuroscience Methods, vol. 161, no. 1, pp. 62-74, 2007.

[5] K. Inoue, F. Kato, and S. Lee, "Haptic device using flexible sheet and air jet for presenting virtual lumps under skin," in Proc. IEEE/RSJ Int'l Conference on Intelligent Robots and Systems, 2009, pp. 1749-1754.

[6] T. E. Faber, Fluid dynamics for physicists. United Kingdom: Cambridge University Press, 1995.

[7] G. A. Gescheider, Psychophysics: Method, Theory, and Application. Hillsdale, NJ: Lawrence Erlbaum Associates, Inc., 1985.

[8] F. A. Wichmann and N. J. Hill, "The psychometric function: I. Fitting, sampling, and goodness of fit." Perception and Psychophysics., vol. 63(8), pp. 1293-1313, 2001. 